\title{
The Outcome and Efficacy of Recanalization in Patients with Acute Internal Carotid Artery Occlusion
}

\author{
J.H. Kwak, L. Zhao, J.K. Kim, S. Park, D.-g. Lee, J.H. Shim, D.H. Lee, J.S. Kim, and D.C. Suh
}

\begin{abstract}
BACKGROUND AND PURPOSE: Acute occlusion of the ICA is often associated with poor outcomes and severe neurologic deficits. This study was conducted to evaluate outcome of the occluded ICA and efficacy of recanalization under protective flow arrest.

MATERIALS AND METHODS: Fifty consecutive patients who underwent endovascular treatment for acute ICA occlusion were identified from the prospectively collected data base. We assessed NIHSS $_{0}$, occlusion type (cardioembolism vs atherosclerosis), occlusion level (supraclinoid-terminal, petrocavernous, or bulb-cervical), recanalization degree (TICI), and efficacy of recanalization (protective flow arrest vs nonprotection) leading to better outcome.

RESULTS: Successful recanalization $(\mathrm{TICl} \geq 2$ ) was obtained in $90 \%$ of patients and good recovery (mRS $\leq 2)$ in $60 \%$ of patients. Good outcome was related to National Institutes of Health Stroke Scale score on admission $(P<.001), \mathrm{TICl}(P<.007)$, occlusion type $(P=.022)$, and occlusion level $(P=$.038). Poor initial patient status, less recanalization, cardioembolism, and supraclinoid-terminal occlusion were associated with poor prognosis. Application of protective flow arrest led to better outcome in the distal ICA segment than in the bulb-cervical segment.
\end{abstract}

CONCLUSIONS: In addition to the initial patient status and successful recanalization, the occlusion level or type of the occluded ICA could affect clinical outcome. In this study, treatment benefits of protective flow arrest were accentuated in patients with ICA occlusion above the bulb-cervical segment.

ABBREVIATIONS: ECIC = extracranial-intracranial; IMS III = Interventional Management of Stroke III trial; NIHSS $=$ National Institutes of Health Stroke Scale score on admission; TOAST $=$ Trial of Org 10172 in Acute Stroke Treatment

A cute occlusion of the ICA is often associated with poor outcomes and severe neurologic deficits. ${ }^{1,2}$ Acute ICA occlusions are more resistant than MCA occlusions to administration of intravenous tPA. ${ }^{3,4}$ In the Trial of Org 10172 in Acute Stroke Treatment (TOAST), 10\% of patients were diagnosed with ICA occlusion, which resulted in neurologic disability in $40 \%$ and mortality in $20 \%$ of patients. ${ }^{5}$

Intra-arterial mechanical thrombectomy has been increas-

Received May 20, 2013; accepted after revision August 12.

From the Department of Radiology and Research Institute of Radiology (J.H.K., L.Z., S.P., D.-g.L., J.H.S., D.H.L., D.C.S.) and Department of Neurology (J.S.K.), University of Ulsan, College of Medicine, Asan Medical Center, Seoul, Korea; Department of Radiology (J.H.K.), Daegu Fatima Hospital, Daegu, Korea; Department of Radiology (L.Z.), First Affiliated Hospital of Nanjing Medical University, Nanjing, China; and Department of Radiology (J.K.K.), Chung-Ang University, College of Medicine, Seoul, Korea.

Please address correspondence to Dae Chul Suh, MD, PhD, Department of Radiology, Asan Medical Center, University of Ulsan, College of Medicine, 86 Asanbyeongwon-Gil, Songpa-Gu, Seoul 138-736, Korea; e-mail: dcsuh@amc.seoul.kr

http://dx.doi.org/10.3174/ajnr.A3747

ingly used in the management of acute ischemic stroke with the recent introduction of stent retrievers (nondetachable microcatheter-based stentlike devices). ${ }^{6-9}$ Despite anecdotal endovascular attempts, an effective management approach has not been well established for patients with acute ICA occlusion that is not applicable to intravenous and/or intra-arterial thrombolysis. ${ }^{10-13}$ The aims of the present study were to investigate the outcome and factors associated with recanalization therapy in acute occlusion of the ICA and to assess the efficacy of proximal flow arrest.

\section{MATERIALS AND METHODS}

Between April 2007 and November 2011, intra-arterial recanalization therapy was performed on 50 consecutive patients who met the following criteria: 1) ICA occlusion presented with acute stroke, and 2) a small early infarct area ( $<$ one-third of vascular territory) on a DWI, with mismatched perfusion-diffusion. MR imaging-specific perfusion-diffusion mismatch was identified as having a mismatch larger than $20 \%$ measured visually from the relative mean transit time map and DWI. ${ }^{14}$ Patients with ICA 
Table 1: Baseline characteristics of enrolled patients, subdivided depending on outcome

\begin{tabular}{|c|c|c|c|c|}
\hline Parameter & Good $(m R S \leq 2) n=30$ & Poor $(m R S>2) n=20$ & Total $n=50$ & $P$ Value \\
\hline $\mathrm{Age}^{\mathrm{a}} \mathrm{y}$ & 68 (56-71) & $67(56-77)$ & $68(56-72)$ & $.684^{\mathrm{b}}$ \\
\hline Sex, M/F & $23 / 7$ & $10 / 10$ & $33 / 17$ & $.051^{\mathrm{c}}$ \\
\hline \multicolumn{5}{|l|}{ Risk factors } \\
\hline Hypertension & 19 & 10 & 29 & $.349^{c}$ \\
\hline Diabetes mellitus & 9 & 9 & 18 & $.279^{c}$ \\
\hline Hyperlipidemia & 10 & 6 & 16 & $.804^{\mathrm{c}}$ \\
\hline Cardiac disease & 9 & 11 & 20 & $.077^{c}$ \\
\hline Previous stroke & 3 & 7 & 10 & $.067^{\mathrm{c}}$ \\
\hline Family history & 4 & 6 & 10 & $.171^{\mathrm{c}}$ \\
\hline Smoking & 11 & 5 & 16 & $.386^{\mathrm{c}}$ \\
\hline Alcohol & 14 & 8 & 22 & $.642^{c}$ \\
\hline Onset & & & & $.063^{\mathrm{c}}$ \\
\hline$<6$ hours & 10 & 12 & 22 & \\
\hline$\geq 6$ hours & 20 & 8 & 28 & \\
\hline $\mathrm{NIHSS}_{0}{ }^{\mathrm{a}}$ & $6(3-11)$ & $15(10-18)$ & $10(4-15)$ & $<.001^{\mathrm{b}}$ \\
\hline Occlusion level & & & & $.038^{\mathrm{c}}$ \\
\hline Supraclinoid-terminal & 6 & 8 & 14 & \\
\hline Petrocavernous & 9 & 9 & 18 & \\
\hline Bulb-cervical & 15 & 3 & 18 & \\
\hline Occlusion type & & & & $.022^{c}$ \\
\hline Cardioembolism & 7 & 11 & 18 & \\
\hline Large-artery disease & 23 & 9 & 32 & \\
\hline Flow arrest & & & & $.419^{c}$ \\
\hline Yes & 17 & 9 & 26 & \\
\hline No & 13 & 11 & 24 & \\
\hline $\mathrm{TICl}$ & & & & $.007^{\mathrm{c}}$ \\
\hline$\geq 2$ & 30 & 15 & 45 & \\
\hline$<2$ & 0 & 5 & 5 & \\
\hline
\end{tabular}

${ }^{a}$ Data expressed as median (interquartile range).

${ }^{b}$ Mann-Whitney test.

${ }^{\mathrm{c}}$ The $\chi^{2}$ test or the Fisher exact test.

occlusion with a history of transient ischemic attack or previous stroke in the subacute or chronic stages, intracranial occlusion beyond the ICA, or occlusion of the posterior circulation were excluded. Our institutional review board approved the design of the study and the use of clinical data, and all patients provided written informed consent.

Table 1 shows the clinical and angiographic data of patients with ICA occlusion. The following variables that may affect the rate of adverse events were included as possible risk factors: age; sex (men vs women); presence of vascular risk factors (hypertension, diabetes mellitus, hyperlipidemia, cardiac disease, stroke history, family history, smoking, and alcohol); symptom onset; NIHSS score on admission (NIHSS $_{\mathrm{o}}$ ); occlusion level and type; application of protective transient flow arrest; and postprocedural TICI grades at the site of occlusion after revascularization.

Protective flow arrest with a proximal or distal protection balloon or manual neck vessel compression was applied at our institute. Various thrombectomy attempts, including aspiration or retrieval of an intravascular clot, were performed under proximal or distal balloon protection. We used a neck compression technique $(n=3)$ before a proximal protective balloon became available in our medical institute. The ipsilateral neck was manually compressed in the area of the carotid arterial pulsation or on the palpable guiding catheter in the carotid artery while aspiration was performed via a guiding catheter positioned in the ICA. Aspiration was done by applying continuous negative pressure by using a 30-cc syringe for approximately $10-15$ seconds and was repeated 2 or 3 times during an approximately 30 -second interval. $^{15}$
Application of the protective flow arrest technique was added as a factor to the outcome analysis.

To analyze the occlusion level (site), we applied the embryologic segmental anatomy of the ICA. ${ }^{16}$ The ICA was divided by the embryologic vascular remnants that can help identify the occlusion level and work as collateral channels, leading to subsequent embolism (Fig 1). The 50 patients were categorized according to occlusion level, based on the 3 most commonly encountered anatomic parts in the ICA segments: supraclinoid-terminal $(n=14)$, petrocavernous $(n=18)$, and bulb-cervical $(n=18)$.

Patients with acute stroke who presented with symptom onset of $<6$ hours ( $n=22)$ were treated according to our institution's protocol by intravenous and/or intra-arterial thrombolytic therapies. ${ }^{17} \mathrm{~A}$ total of 7 patients received IV tPA and subsequently underwent endovascular recanalization because there was no improvement in their status. The patients who presented with acute symptom onset of $\geq 6$ hours ( $n=28$ ) were considered for recanalization on the basis of the extent of the infarct and diffusion-perfusion/symptom mismatch after discussion with neurologists. In those patients with low initial NIHSS scores, the recanalization procedure in our study was performed based on progressive or fluctuating symptoms $(n=17)$, or because of perfusion abnormality $(n=24)$.

The subtype classification of large-artery atherosclerosis ( $n=$ $32)$ or cardioembolism $(n=18)$ for ICA occlusion was based on the modified TOAST classification at the time of admission. ${ }^{5}$ The pretherapeutic neurologic status of the patient was evaluated by NIHSS $_{\mathrm{o}}$. The mRS was used to assess the final clinical outcome at 6 months (good outcome, mRS $\leq 2$; poor outcome, $\mathrm{mRS} \geq 3$ ).

\section{Recanalization Procedure}

Fifty patients with occlusion of the ICA were treated with suction, angioplasty, and/or stent placement in conjunction with retrieval devices. All patients were given $200 \mathrm{mg}$ of aspirin and a loading dose of $300 \mathrm{mg}$ of clopidogrel before the procedure, if they were not already taking these medications. After the procedure, $100 \mathrm{mg}$ of aspirin once daily was continued as a permanent medication. In addition, $75 \mathrm{mg}$ of clopidogrel was given once daily for at least 6 months after the procedure. ${ }^{18}$

The procedural details were the same as we had described previously, except that a $9 \mathrm{~F}$ sheath was used for the proximal protection balloon catheter. ${ }^{15,18-23}$ Protective flow arrest methods included proximal balloon protection with or without distal balloon protection or manual neck compression. The distal balloon was introduced through a $4 \mathrm{~F}$ catheter or the occluded segment after application of the proximal balloon. 

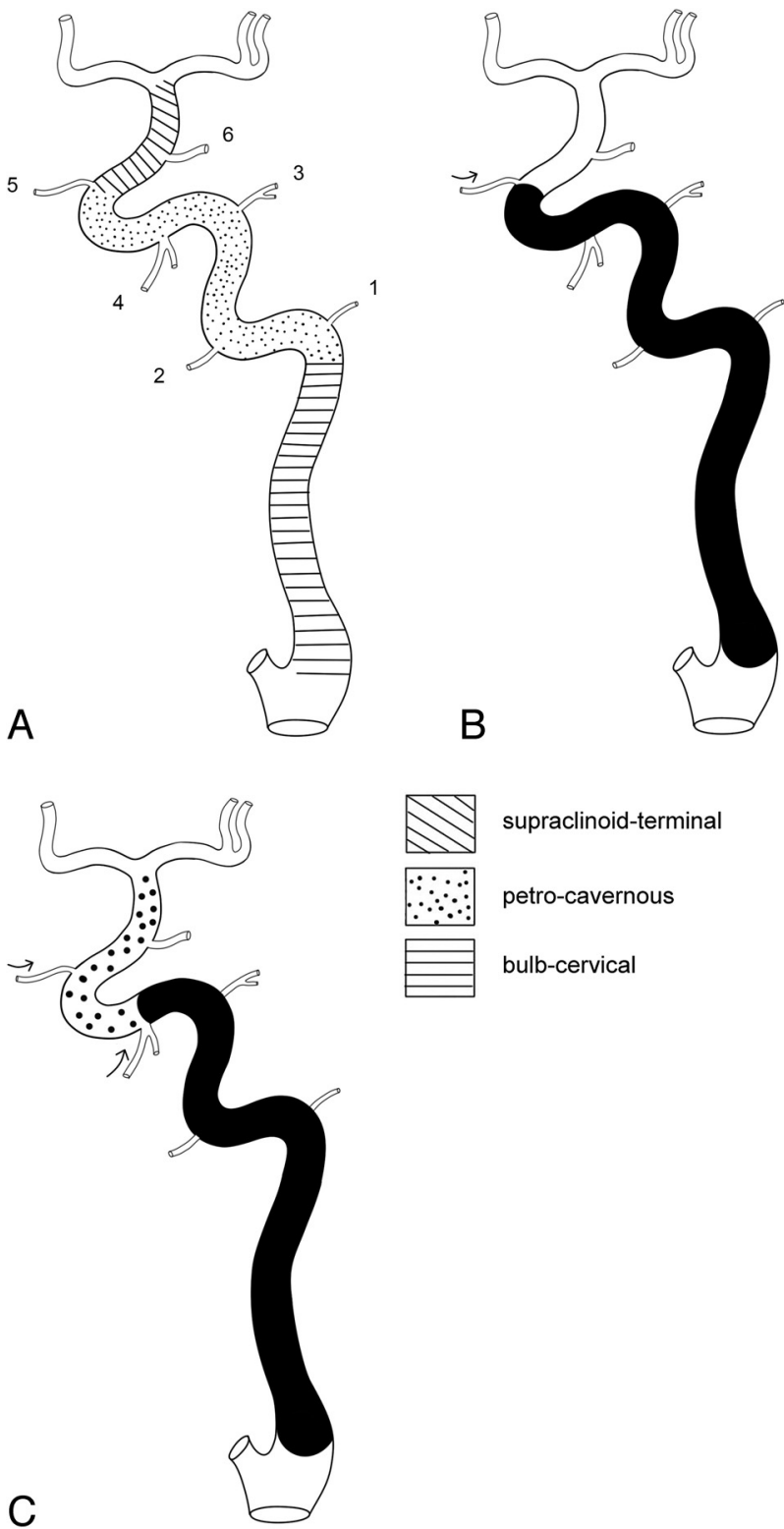

FIG 1. A, Division of the ICA segments into 3 parts based on embryologic classification ( 1 = caroticotympanic artery, 2 = mandibular artery, $3=$ meningohypophyseal trunk, $4=$ inferolateral trunk, $5=$ ophthalmic artery, $6=$ posterior communicating artery). When there is occlusion in the ICA (B), each branch between the segments may develop as a collateral, leading to thromboembolism by reopening the occluded vessel (C). Arrows in $B$ and $C$ are the direction of the flow. The inferolateral trunk plays a role in reopening the occluded the ICA segment and generating distal emboli.

TICI perfusion categories include grade 0 (no perfusion), grade I (penetration with minimal perfusion), grade II (partial perfusion), grade IIa (only partial filling; $<$ two-thirds of the entire vascular territory), grade IIb (complete but slow filling of all of the expected vascular territory), and grade III (complete filling). ${ }^{24}$ Although several studies have defined successful reperfusion as TICI IIb or greater, they used various cutoff values (50\% vs $67 \%$ ) for grade IIb, thus rendering comparisons across studies difficult or impossible. $^{25}$ Therefore, we defined TICI grade II-III as successful reperfusion. ${ }^{25}$

\section{Basic Strategy of Recanalization According to Occlusion Levels}

Proximal ICA Occlusion (Bulb-Cervical Segment). If there was an insufficient length of stump of the carotid bulb portion or if suction by proximal balloon catheter (eg, Optimo; Tokai Medical Products, Aichi, Japan) was ineffective, then a $4 \mathrm{~F}$ catheter was passed along the 0.035 -inch guidewire through the occluded segment and a distal balloon (eg, PercuSurge; Medtronic, Minneapolis, Minnesota) was introduced as shown in Fig $2 A$. Retrograde collateral filling of the segmental ICA anatomy as shown in Fig 1 was used as a guide for the subsequent procedure.

The location of the $4 \mathrm{~F}$ catheter tip in the free lumen was identified by the suction of thrombotic debris in the distal segment beyond the occlusion and the regurgitation of blood. A protective distal balloon was introduced via the $4 \mathrm{~F}$ catheter. Once protective distal occlusion was achieved, suction of the proximal ICA segment was followed by angioplasty and/or stent placement if there was any significant stenotic lesion. The ICA was irrigated by saline into the external carotid artery before balloon deflation to ensure that there was no debris in the ICA.

Distal ICA Occlusion (Petrocavernous Segment). Suction was applied via the proximal balloon catheter that was introduced in the cervical segment of the ICA (Fig $2 B$ ). If the suction was ineffective, then further clot retrieval was attempted by a Solitaire stent (Covidien, Irvine, California) or the Penumbra system (Penumbra, Alameda, California). If atherosclerotic stenosis remained after clot removal, then subsequent angioplasty and/or stent placement was performed under proximal balloon protection.

Occlusion of the Supraclinoid-Terminal Segment and/or an Intracranial Vessel. As was done with distal ICA occlusion, suction was applied via the proximal balloon catheter introduced in the cervical segment of the ICA. For residual intracranial vessel occlusion, further clot retrieval was attempted with a Solitaire or Penumbra catheter under proximal balloon protection (Fig 2C).

\section{Statistical Analysis}

We performed univariate analysis by using the $\chi^{2}$ test or the Fisher exact test to assess the relationship between outcome and incontinuous independent variables. Mann-Whitney tests were used for continuous variables including age and NIHSS $_{\mathrm{o}}$. Stratification analysis with the Cochran-Mantel-Haenszel test was used to assess the significant relationship between the potential risk factor and outcome, when there was a suspected confounding effect of another factor. All reported probability values were 2 -sided, and $P$ values of $<.05$ were considered statistically significant. We performed all statistical analyses by using SAS version 8.1 (SAS Institute, Cary, North Carolina).

\section{RESULTS}

Successful recanalization (TICI $\geq 2$ ) was obtained in 45 (90\%) of 50 patients, and good outcome ( $\mathrm{mRS} \leq 2)$ at 6 months was obtained in $30(60 \%)$ of 50 patients. Good outcome was related to NIHSS $_{\mathrm{o}}(P<.001)$, TICI $(P=.007)$, occlusion type $(P=.022)$, and occlusion level $(P=.038)$ (Table 1). A lower NIHSS $_{\mathrm{o}}$ and successful recanalization were significantly correlated with a good clinical outcome. Supraclinoid-terminal occlusion showed 


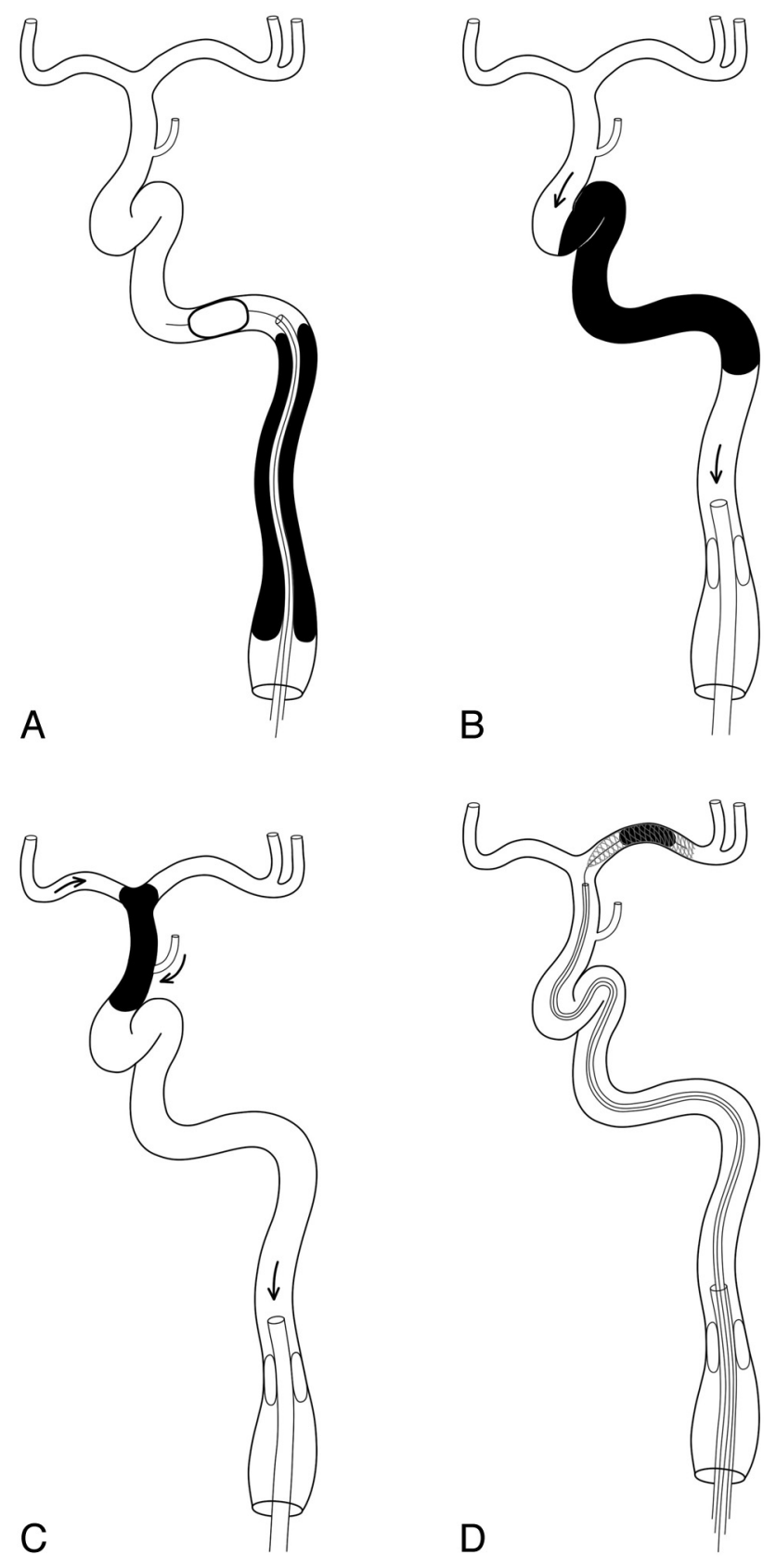

FIG 2. Schematic diagrams showing the protective flow arrest methods in each occluded level. A, A distal balloon was deployed beyond the bulb-cervical occlusion through a $4 \mathrm{~F}$ catheter. Proximal balloons may be used together. Proximal flow arrest may be achieved by a proximal balloon catheter for petrocavernous $(B)$ and supraclinoid-terminal $(C)$ occlusions. Arrows in $B$ and $C$ are the flow direction when a negative pressure is applied by aspiration. There are 2 sources (the $\mathrm{Al}$ and the posterior communicating artery) of the expected collateral channel in the supraclinoid-terminal segment. A fragmented or additional clot in the intracranial artery is subsequently retrieved by a stent retriever under proximal balloon protection $(D)$.

poorer prognosis than petrocavernous or bulb-cervical occlusion. Results of stratification analysis (Table 2) revealed a significant correlation between application of flow arrest and clinical outcome with stratification according to occlusion levels $(P=.049)$, that is, the application of protective flow arrest led to a better outcome in the distal ICA segment occlusion. On the other hand,
Table 2: Analysis of the relationship between application of protective flow arrest and clinical outcome with stratification according to occlusion levels

\begin{tabular}{lccccc}
\hline & \multicolumn{2}{c}{ Bulb $(n=18)$} & & \multicolumn{2}{c}{ Above Bulb $(n=32)$} \\
\cline { 2 - 3 } & $\begin{array}{c}\text { With Flow } \\
\text { Arrest }\end{array}$ & $\begin{array}{c}\text { Without } \\
\text { Flow Arrest }\end{array}$ & & $\begin{array}{c}\text { With Flow } \\
\text { Arrest }\end{array}$ & $\begin{array}{c}\text { Without } \\
\text { Flow Arrest }\end{array}$ \\
\hline $6 \mathrm{~m} \mathrm{mRS} \leq 2$ & 4 & 11 & & 13 & 2 \\
$6 \mathrm{~m} \mathrm{mRS}>2$ & 1 & 2 & & 8 & 9 \\
\hline
\end{tabular}

Note:-The results of the Cochran-Mantel-Haenszel test and Cochran $\chi^{2}$ value of $3.89(P=.049)$ further verify a relationship between flow arrest and clinical outcome.

${ }^{a}$ Above bulb includes supraclinoid-terminal and petrocavernous segments.

outcome was irrelevant to the application of protective flow arrest in the bulb-cervical segment occlusion.

Five patients $(10 \%)$ who had a failed recanalization $($ TICI $<2)$ showed poor clinical outcomes $(\mathrm{mRS}>2)$ at 6 months. Patients with acute stroke (onset time $<6$ hours) with ICA occlusion showed relatively poor clinical outcomes $(55 \% ; 12 / 22)$, despite relatively high recanalization rates (TICI $\geq 2 ; 82 \% ; 18 / 22$ ). Of the 22 patients who had ICA occlusion with onset time $<6$ hours, 10 patients $(45 \%)$ had a good clinical outcome and 5 patients $(23 \%)$ died. These results were partially related to the fact that $17(77 \%)$ of 22 patients with early onset (onset time $<6$ hours) had a high NIHSS score $(>10)$ compared with $6(21 \%)$ of 28 patients with an onset time $\geq 6$ hours $(P=.000)$.

Thirty-four (94\%) of 36 patients with bulb-cervical or petrocavernous ICA occlusion showed successful recanalization. In contrast, 11 (79\%) of 14 patients with supraclinoid-terminal occlusion of the ICA showed successful recanalization, resulting in only $6(43 \%)$ of 14 patients with a good outcome at 6 months. The initial patient status was poorer in cases of cardioembolism than in cases of large-artery disease (Fig 3). Large-artery disease was more common in the bulb-cervical segment compared with cardioembolism, which was more common in the supraclinoid-terminal segment of the ICA.

There were 3 patients (6\%) with postprocedural symptomatic intracerebral hemorrhage. Six patients with intracerebral hemorrhage $(12 \%)$ were asymptomatic. Two of the hemorrhages were related to massive infarcts. One was a massive hemorrhage that led to death. Of the 50 patients, $6(12 \%)$ died within 6 months after the procedure. The cause of death was related to massive infarction $(n=4)$, intracranial hemorrhage $(n=1)$, or pneumonia-induced sepsis $(n=1)$.

\section{DISCUSSION}

Our study revealed that successful recanalization (TICI $\geq 2$ ) was achieved in $45(90 \%)$ of 50 patients and resulted in a good outcome (mRS > 2) after 6 months in $30(60 \%)$ of 50 patients with acute ICA occlusion. Jovin et $\mathrm{al}^{26}$ reported a favorable 30-day outcome $(\mathrm{mRS} \leq 2)$ with endovascular treatment in $5(33 \%)$ of 15 patients with acute ICA occlusion and $7(88 \%)$ of 8 patients with subacute ICA occlusion. Using combined mechanical recanalization with aspiration and a stent retriever, Papanagiotou et $\mathrm{al}^{10}$ obtained successful recanalization in $14(63 \%)$ of 22 patients and a good outcome at 3 months in $13(59 \%)$ of 22 patients. Although there are few large series of recanalization for patients with acute ICA occlusion, our results showed the possibility of an improved recanalization rate and good outcome among these patients. ${ }^{22}$ Although the natural history of acute stroke with a pro- 
Large artery disease

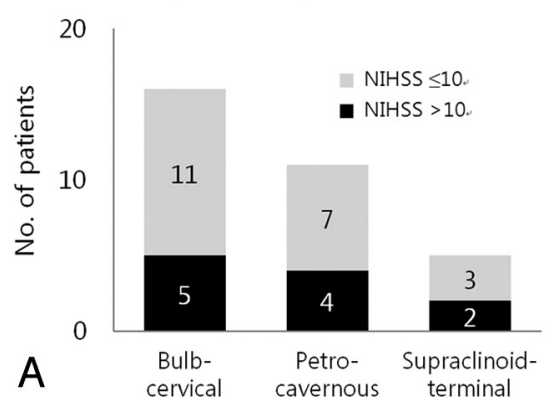

\section{Cardioembolism}

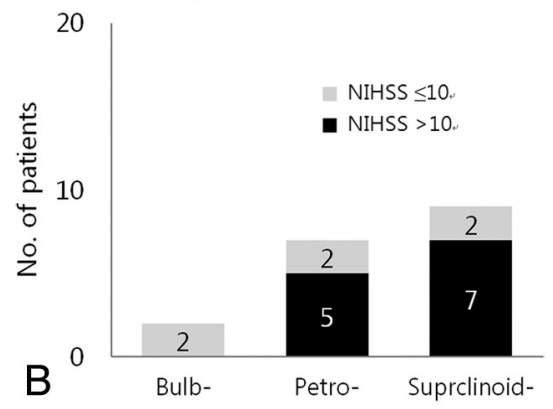

FIG 3. Different proportions of initial patient status according to the occlusion level based on the occlusion type (large-artery disease vs cardioembolism). There was a reversed trend of patient number for each occlusion type according to the lesion level. The initial status of patients with cardioembolism in the supraclinoid-terminal segment of the ICA was proportionally poorer than that of patients with large-artery disease.

gressive or fluctuating symptom pattern or perfusion abnormality in patients with low NIHSS is not completely known, recanalization may be warranted because mild or improving stroke may have poor short-term outcomes, ${ }^{27}$ and acute ischemic stroke from occlusion of the ICA is one of the most devastating forms of acute ischemia, with only $2 \%-30 \%$ of patients achieving good recovery. ${ }^{28}$

Good outcome was related to initial patient status, postprocedural TICI grade, occlusion type, and occlusion level. The more favorable outcome may be related to good collateral circulation and small ischemic core or large penumbra lesion, because retrograde ICA filling, ophthalmic collaterals, or leptomeningeal collaterals are related to better short-term outcomes. ${ }^{22,29,30}$ Assessment of occlusion level based on the segmental ICA anatomy as in our study could provide better anatomic confinement and allows the application of proximal and/or distal protection with flow arrest, which subsequently could play a role in improving the recanalization rate and resulting in good clinical outcome. ${ }^{9,10}$ The rate of symptomatic intracerebral hemorrhage seen in our study was $6 \%$, which was similar to $6.2 \%$ in the Interventional Management of Stroke III (IMS III) trial and 6\% of fatal and nonfatal symptomatic intracranial hemorrhage in SYNTHESIS. ${ }^{31,32}$

Although the IMS III trial did not show any difference in functional independence ( $\mathrm{mRS} \leq 2)$ with endovascular therapy after intravenous tPA, compared with intravenous tPA alone, the ICA occlusion subgroup in the trial revealed the difference; the rate of partial or complete recanalization at 24 hours, as seen on CT angiograms at both baseline and at 24 hours, was $81 \%$ for an occlusion in the ICA in the endovascular therapy group, compared with $35 \%$ for an occlusion in the ICA in the intravenous tPA group. ${ }^{32}$ In addition, the segmental location of the ICA occlusion and the mechanism of occlusion (cardioembolism vs atherosclerosis) were not specified in the IMS III and SYNTHESIS trials. ${ }^{31,32}$ The MR RESCUE (Mechanical Retrieval and Recanalization of Stroke Clots Using Embolectomy) trial studied patients with NIHSS scores of 6-29 who had a large-vessel, anterior-circulation ischemic stroke and who were randomly assigned within 8 hours after the onset of symptoms to undergo either mechanical embolectomy or standard medical care. ${ }^{33}$ However, the study used only the old version of recanalization devices (Merci [Concentric Medical, Mountain View, California] or Penumbra)

without using Trevo (Stryker, Kalamazoo, Michigan) or Solitaire, both of which have revealed a better primary efficacy (61\%) outcome (ie, successful recanalization without symptomatic intracranial hemorrhage) vs $21 \%$ with Merci. ${ }^{34}$

Because there is no standardized recanalization technique for ICA occlusion in patients with acute stroke, mechanical and/or intra-arterial thrombolysis for intracranial occlusion has been followed by angioplasty and/or stent placement, aspiration thrombectomy, or clot retrieval with various devices. ${ }^{9-12,35}$ Among the factors affecting good outcome, the effect of revascularization (postprocedural TICI grade) may depend on the methodology used to improve the final luminal patency for each occlusion type and level. Among those methods, protective flow arrest applies simple suction that effectively aspirates the clot and prevents distal migration to the intracranial vessels during clot retrieval. ${ }^{36}$ The angioplasty and/or stent placement performed in our study can be part of the recanalization procedure if the stent retriever is not effective and the indications can differ from those applied in SAMMPRIS (Stenting vs Aggressive Medical Management for Preventing Recurrent Stroke in Intracranial Stenosis), which included patients with TIA or nonsevere stroke occurring within 30 days of their enrollment and attributed to $70 \%-99 \%$ stenosis of a major intracranial artery. ${ }^{37}$

Because there is little stump for proper balloon engagement in bulb-cervical occlusion, a combination of proximal and distal protective balloons (as shown in Fig 2) may provide more protection against additional thromboemboli during the recanalization procedure. For petrocavernous and supraclinoid-terminal segment occlusion, proximal flow arrest alone may be applied to aspirate the clot and prevent distal thromboembolism during clot retrieval. The flow reversal technique or even manual neck compression may also be effective for protective proximal flow arrest. ${ }^{9,12}$ Simple suction thrombectomy can be applied by using a proximal protection balloon with which the stent retriever is subsequently used.

Extracranial-intracranial (ECIC) bypass surgery in patients with symptomatic occlusion of the ICA was illustrated by a new operating technique and patients without naturally occurring collateral flow to maintain adequate circulation might benefit from ECIC bypass surgery. Recently, ECIC bypass surgery plus medical therapy was not found to reduce the risk for recurrent ipsilateral ischemic stroke compared with medical therapy alone. ${ }^{38}$ ECIC bypass surgery may have limitations regarding response to a recent, symptomatic occlusion containing remarkable salvageable brain parenchyma at the time of symptom presentation. ${ }^{39}$

Our study had several limitations. First, the overall number of our study patients was relatively small; therefore, further analysis by categorization into small subgroups made it difficult to draw any definite conclusions. Second, the procedural methodology 
used in our study was not homogeneous because various thrombectomy attempts, including aspiration or retrieval of an intravascular clot, were performed with or without proximal or distal balloon protection. Despite the use of currently available management strategies, the clinical and angiographic responses to therapy remain limited. ${ }^{6}$ Therefore, further data must be added to support our conclusion, though it is difficult to perform a randomized controlled study because acute ICA occlusions presenting with severe stroke symptoms are relatively rare, even at highvolume stroke centers.

\section{CONCLUSIONS}

Our results showed that successful recanalization and a good outcome could be achieved by combined mechanical thrombectomy, especially under protective flow arrest. Good outcome was related to initial patient status, postprocedural TICI grade, occlusion type, and occlusion level. Our study also revealed that the application of proximal and/or distal protection with flow arrest helps to improve the recanalization rate and results in a good clinical outcome.

Disclosures: Deok Hee Lee-UNRELATED: Board Membership: Covidien, Comments: Asian clinical advisory board member; Payment for Lectures (including service on speaker bureaus): Codman; Patents (planned, pending, or issued): S\&G Biotech; Royalties: S\&G Biotech.

\section{REFERENCES}

1. Seet RC, Wijdicks EF, Rabinstein AA. Stroke from acute cervical internal carotid artery occlusion: treatment results and predictors of outcome. Arch Neurol 2012:1-6

2. Paciaroni M, Balucani C, Agnelli G, et al. Systemic thrombolysis in patients with acute ischemic stroke and Internal Carotid ARtery Occlusion: the ICARO study. Stroke 2012;43:125-30

3. Jansen O, von Kummer R, Forsting M, et al. Thrombolytic therapy in acute occlusion of the intracranial internal carotid artery bifurcation. AJNR Am J Neuroradiol 1995;16:1977-86

4. Linfante I, Llinas RH, Selim M, et al. Clinical and vascular outcome in internal carotid artery versus middle cerebral artery occlusions after intravenous tissue plasminogen activator. Stroke 2002;33:2066-71

5. Adams HP, Jr., Bendixen BH, Kappelle LJ, et al. Classification of subtype of acute ischemic stroke. Definitions for use in a multicenter clinical trial. TOAST. Trial of Org 10172 in Acute Stroke Treatment. Stroke 1993;24:35-41

6. Pierot L, van der Bom IM, Wakhloo AK. Advances in stroke: advances in interventional neuroradiology. Stroke 2012;43:310-13

7. Brinjikji W, Rabinstein AA, Kallmes DF, et al. Patient outcomes with endovascular embolectomy therapy for acute ischemic stroke: a study of the National Inpatient Sample: 2006 to 2008. Stroke 2011;42:1648-52

8. Costalat V, Machi P, Lobotesis K, et al. Rescue, combined, and standalone thrombectomy in the management of large vessel occlusion stroke using the Solitaire device: a prospective 50-patient singlecenter study: timing, safety, and efficacy. Stroke 2011;42:1929-35

9. Eesa M, Almekhlafi MA, Mitha AP, et al. Manual aspiration thrombectomy through balloon-tipped guide catheter for rapid clot burden reduction in endovascular therapy for ICA L/T occlusion. Neuroradiology 2012;54:1261-65

10. Papanagiotou $\mathrm{P}$, Roth $\mathrm{C}$, Walter $\mathrm{S}$, et al. Carotid artery stenting in acute stroke. J Am Coll Cardiol 2011;58:2363-69

11. Hauck EF, Natarajan SK, Ohta H, et al. Emergent endovascular recanalization for cervical internal carotid artery occlusion in patients presenting with acute stroke. Neurosurgery 2011;69:899-907

12. Terada T, Okada H, Nanto M, et al. Endovascular recanalization of the completely occluded internal carotid artery using a flow rever- sal system at the subacute to chronic stage. J Neurosurg 2010;112:563-71

13. Thomas AJ, Gupta R, Tayal AH, et al. Stenting and angioplasty of the symptomatic chronically occluded carotid artery. AJNR Am J Neuroradiol 2007;28:168-71

14. Kang DW, Sohn SI, Hong KS, et al. Reperfusion Therapy in UnclearOnset Stroke Based on MRI Evaluation (RESTORE): a prospective multicenter study. Stroke 2012;43:3278-83

15. Xu GF, Suh DC, Choi CG, et al. Aspiration thrombectomy of acute complete carotid bulb occlusion. J Vasc Interv Radiol 2005;16: $539-42$

16. Lasjaunias P, Berenstein A, Ter Brugge KG. Skull base and maxillofacial region. In: Surgical Neuro-Angiography, 2nd ed. Berlin: Springer-Verlag; 2001:261-90

17. Cho KH, Lee DH, Kwon SU, et al. Factors and outcomes associated with recanalization timing after thrombolysis. Cerebrovasc Dis 2012;33:255-61

18. Choi JW, Kim JK, Choi BS, et al. Adjuvant revascularization of intracranial artery occlusion with angioplasty and/or stenting. $\mathrm{Neu}$ roradiology 2009;51:33-43

19. Liu S, Jung JH, Kim SM, et al. Simultaneous bilateral carotid stenting in high-risk patients. AJNR Am J Neuroradiol 2010;31:1113-17

20. In HS, Lee HY, Park JY, et al. Intracranial stenting in patients with atherosclerotic stenosis associated with various aneurysms in the same diseased arterial segment. AJNR Am J Neuroradiol 2010;31:1895-98

21. Suh DC, Kim JK, Choi JW, et al. Intracranial stenting of severe symptomatic intracranial stenosis: results of 100 consecutive patients. AJNR Am J Neuroradiol 2008;29:781-85

22. Suh DC, Kim JK, Choi CG, et al. Prognostic factors for neurologic outcome after endovascular revascularization of acute symptomatic occlusion of the internal carotid artery. AJNR Am J Neuroradiol 2007;28:1167-71

23. Pyun HW, Suh DC, Kim JK, et al. Concomitant multiple revascularizations in supra-aortic arteries: short-term results in $\mathbf{5 0}$ patients. AJNR Am J Neuroradiol 2007;28:1895-901

24. Higashida RT, Furlan AJ, Roberts $H$, et al. Trial design and reporting standards for intra-arterial cerebral thrombolysis for acute ischemic stroke. Stroke 2003;34:e109-37

25. Suh SH, Cloft HJ, Fugate JE, et al. Clarifying differences among Thrombolysis in Cerebral Infarction scale variants: Is the artery half open or half closed? Stroke 2013;44:1166-68

26. Jovin TG, Gupta R, Uchino K, et al. Emergent stenting of extracranial internal carotid artery occlusion in acute stroke has a high revascularization rate. Stroke 2005;36:2426-30

27. Smith EE, Fonarow GC, Reeves MJ, et al. Outcomes in mild or rapidly improving stroke not treated with intravenous recombinant tissue-type plasminogen activator: findings from Get With The Guidelines-Stroke. Stroke 2011;42:3110-15

28. Fischer U, Mono ML, Schroth G, et al. Endovascular therapy in 201 patients with acute symptomatic occlusion of the internal carotid artery. Eur J Neurol 2013;20:1017-24, e87

29. Choi JW, Kim JK, Choi BS, et al. Angiographic pattern of symptomatic severe M1 stenosis: comparison with presenting symptoms, infarct patterns, perfusion status, and outcome after recanalization. Cerebrovasc Dis 2010;29:297-303

30. Liebeskind DS, Cotsonis GA, Saver JL, et al. Collateral circulation in symptomatic intracranial atherosclerosis. J Cereb Blood Flow Metab 2011;31:1293-301

31. Ciccone A, Valvassori L, Nichelatti M, et al. Endovascular treatment for acute ischemic stroke. $N$ Engl J Med 2013;368:904-13

32. Broderick JP, Palesch YY, Demchuk AM, et al. Endovascular therapy after intravenous t-PA versus t-PA alone for stroke. $N$ Engl J Med 2013;368:893-903

33. Kidwell CS, Jahan R, Gornbein J, et al. A trial of imaging selection and endovascular treatment for ischemic stroke. $N$ Engl J Med 2013;368:914-23

34. Saver JL, Jahan R, Levy EI, et al. Solitaire flow restoration device 
versus the Merci retriever in patients with acute ischaemic stroke (SWIFT): a randomised, parallel-group, non-inferiority trial. Lancet 2012;380:1241-49

35. Lü PH, Park JW, Park S, et al. Intracranial stenting of subacute symptomatic atherosclerotic occlusion versus stenosis. Stroke 2011;42:3470-76

36. Chueh JY, Wakhloo AK, Gounis MJ. Effectiveness of mechanical endovascular thrombectomy in a model system of cerebrovascular occlusion. AJNR Am J Neuroradiol 2012;33:1998-2003

37. Chimowitz MI, Lynn MJ, Turan TN, et al. Design of the stenting and aggressive medical management for preventing recurrent stroke in intracranial stenosis trial. J Stroke Cerebrovasc Dis 2011;20:357-68

38. Powers WJ, Clarke WR, Grubb RL, et al. Extracranial-intracranial bypass surgery for stroke prevention in hemodynamic cerebral ischemia: the Carotid Occlusion Surgery Study randomized trial. JAMA 2011;306:1983-92

39. Rodríguez-Hernández A, Josephson AS, Langer D, et al. Bypass for the prevention of ischemic stroke. World Neurosurg 2011;76(6 Suppl):S72-79 